\title{
Effect of Sex Determining Region Y-Box 2 on the Biological Characteristics of Breast Cancer Cells and its Clinical Significance
}

\author{
LIANJI FENG, JINGMIN WANG ${ }^{1}$, ZHENLING JI ${ }^{1}$, XIAOLI LE² AND HENG ZHANG*
}

Department of General Surgery, Nanjing Lishui People's Hospital, Zhongda Hospital Lishui Branch, Southeast University, ${ }^{1}$ Department of General Surgery, Zhongda Hospital, Southeast University, ${ }^{2}$ Department of Pathology, Nanjing Lishui People's Hospital, Zhongda Hospital Lishui Branch, Southeast University, Nanjing, Jiangsu 211200, China

Feng et al.: Effect of Sex Determining Region Y-Box 2 on the biological characteristics

To investigate the effects of the sex determining region Y-box 2 gene silencing on biological characteristics and the mitogen-activated protein kinase kinase kinase kinase 4 signal pathway of breast cancer cells. Firstly, the expression of sex determining region Y-box 2 in breast cancer was detected by the immunohistochemical method and the relationship with clinicopathological features was analyzed. Then the sex determining region Y-box 2 silencing breast cancer cell line Michigan Cancer Foundation-7 was prepared, and the messenger ribonucleic acid and protein expression levels of sex determining region Y-box 2, c-Jun N-terminal kinase, p53 and tumor necrosis factor-alpha in the mitogen-activated protein kinase kinase kinase kinase 4 pathway were detected by quantitative polymerase chain reaction and western blotting. Cell proliferation, migration, invasion and apoptosis were detected by the cell counting kit-8, scratch test, transwell test, and flow cytometry. After si-sex determining region Y-box 2 transfection, the expression of sex determining region Y-box 2, c-Jun N-terminal kinase, p53 and tumor necrosis factor-alpha decreased, cell growth was inhibited, cell proliferation, migration and invasion decreased significantly, and apoptosis increased, while si-sex determining region Y-box 2+mitogen-activated protein kinase kinase kinase kinase 4 transfection could reverse the above trend. Sex determining region Y-box 2 silencing can activate the mitogen-activated protein kinase kinase kinase kinase 4 signal pathway, promote the proliferation, migration, and invasion of breast cancer cells and inhibit apoptosis.

Key words: Breast cancer, sex determining region Y-box 2, mitogen-activated protein kinase kinase kinase kinase 4 pathway, gene silencing

Breast cancer was one of the most common malignant tumors affecting the women's health, which was a highly heterogeneous tumor ${ }^{[1]}$. In recent years, the incidence of breast cancer has gradually increased. Some scholars have put forward the tumor stem cell hypothesis that the tumors originate from the cells similar to embryonic stem cells (ESCs) that have the ability of self-renewal and differentiation ${ }^{[2]}$. At present, surgical resection was the main treatment of breast cancer, combined with radiotherapy, chemotherapy, endocrine and other treatments. Although these measures have made some progress in the treatment of breast cancer, the therapeutic effect of breast cancer was not very ideal due to the postoperative metastasis, recurrence and chemotherapy resistance. Therefore, it is particularly important to find new effective treatment methods and treatment targets.

Previous studies have shown that the cancer stem cells (CSCs) were the original cell of the tumor and they were more resistant to apoptosis than the differentiated cancer cells ${ }^{[1-3]}$. Promoting apoptosis or inducing CSC differentiation has become a strategy for cancer treatment ${ }^{[4]}$. And the stem cell marker gene sex determining region Y-box 2 (SOX2) has been found to contribute to tumorigenesis and some cancers ${ }^{[5]}$. It was reported that the location of SOX2 was effective in cancer treatment. However, the function and potential mechanism of SOX2 in the apoptosis resistance of cancer cells was still unclear.

*Address for correspondence

E-mail: 2732741326@qq.com 
The activation of p53 and tumor necrosis factoralpha (TNF- $\alpha)$ in the mitogen-activated protein kinases (MAPK) signaling pathway can regulate the inflammatory and apoptotic signal pathways ${ }^{[6,7]}$. In particular, mitogen-activated protein kinase kinase kinase kinase 4 (MAP4K4) in the MAPK signal pathway can activate the cascade of TNF- $\alpha$ and MAPK signal pathways at the same time and the cascade activation of the MAPK signal pathway can regulate the expression of $\mathrm{p} 38$, c-Jun N-terminal kinase (JNK) and extracellular signal-regulated kinase proteins downstream, thus regulating the cell proliferation and apoptosis ${ }^{[8]}$. Some studies have found that the signal pathway involved in MAP4K4 may be that MAP4K4, as a downstream molecule of SOX2 involved in the induction of apoptosis. The silencing SOX 2 can activate the mitochondrial pathway and death receptor pathway regulated by MAP4K4 molecules to participate in the pathway of apoptosis ${ }^{[9]}$. These findings deepen our understanding of the molecular mechanism of SOX2 in the apoptosis and propose new strategies for the treatment of human breast cancer.

Therefore, in this study, the immunohistochemical method was used to analyze the expression and correlation of SOX2 and MAP4K 4 genes in breast cancer. And the molecular regulation of SOX2 and MAP4K4 in breast cancer cell proliferation and apoptosis were explored in vitro cell experiments, thus provide a theoretical basis for the clinical diagnosis, treatment, and prognosis of breast cancer.

\section{MATERIALS AND METHODS}

\section{Pathological tissue sources:}

From January 2017 to July 2020, 60 patients with breast cancer who underwent surgery in the breast armor surgery of our hospital were collected. The patients were diagnosed as breast cancer by pathological examination, the age distribution was 37-79 y old, the average age was $55 \pm 3.21$ y old. According to the standard of diagnosis and treatment of breast cancer in 2011, the general clinical data of patients were collected, including age, sex, tumor size, location, degree of differentiation, depth of invasion, lymph node metastasis, tumor, nodes and metastases (TNM) stage, other clinicopathological data and the normal paracancerous tissues of the same patient $\geq 5 \mathrm{~cm}$ from the tumor margin were taken as the control group. This study was approved by the Ethics Committee of Nanjing Lishui People's Hospital, Zhongda Hospital Lishui Branch, Southeast University.

\section{Cell culturing:}

Human breast cancer cell line MCF-7 was purchased from the Chinese Academy of Sciences (Shanghai, China) and cultured in a DMEM medium containing $10 \%$ fetal bovine serum (FBS) $(50 \mu \mathrm{g} / \mathrm{ml}$ streptomycin and $100 \mu \mathrm{g} / \mathrm{ml}$ penicillin at $37^{\circ}$ provided with $5 \%$ carbon dioxide). According to the instructions of LipofectamineTM 2000, the cellosaurus cell line (EC9706) cells growing to logarithmic phase were inoculated on a 6 -well plate with $5 \times 10^{4}$ cells/well and cultured in an antibiotic-free medium for $24 \mathrm{~h}$. The Lipo2000-small interfering ribonucleic acid (siRNA) complex was prepared and added to the 6-well plate and cultured for $6 \mathrm{~h}$. The medium containing serum was changed and the transfection effect was detected by western blotting (WB) after $48 \mathrm{~h}$ of culturing. Experimental groups including the control group (without transfection of any sequence), negative group (NC group) (transfection of SOX2 negative control sequence), pcDNA-SOX2 group (transfection of SOX2 overexpression vector), small interfering-SOX2 (si-SOX2) group (transfection of si-SOX2) and siSOX2+MAP4K4 group (transfection of silent SOX2 and addition of MAP4K4 signal pathway agonist).

\section{Cell transfection and cloning:}

The cells with good density in each group were selected for cell transfection. Breast cancercells in the logarithmic growth phase were selected for cell counting. 1000 cells per well were inoculated into a six-well plate. 2 $\mathrm{ml}$ culture medium was added to each well and cultured for $7 \mathrm{~d}$. Take out the a six-well plate ( 2 multiple wells in each group) and discard the culture medium in the well, wash it twice with phosphate-buffered saline (PBS) and fix it with methanol for $10 \mathrm{~min}$ and finally dye it with $0.1 \%$ crystal violet for $20 \mathrm{~min}$. After washed with PBS, 5 visual fields were randomly selected to observe the cells under a microscope $(400 \mathrm{x})$ and the colony count was counted.

\section{Cell proliferation:}

The cells in the logarithmic growth phase were selected and made into a single cell suspension and inoculated evenly in a 96-well plate (100 $\mu 1 /$ well, 5000 cells $)$ until the cells adhered to the wall. According to the experimental requirements, the medium was changed after the same amount of trypticase soy agar or tryptone soya agar (TSA) or culture medium was treated for 24 $\mathrm{h}$ and the medium containing $10 \mu \mathrm{l}$ cell counting kit- 8 (CCK-8) solution was added to each well, with a total volume of $100 \mu$ l. The 96 -well plate was shaken evenly 
and incubated for $0.5-4 \mathrm{~h}$. The absorbance of each well at $490 \mathrm{~nm}$ wavelength was measured by the microplate reader.

\section{Cell cycle:}

The cells in the logarithmic growth phase were prepared into a single cell suspension and inoculated in a six-well plate. After the cells adhered to the wall, the drugs were added and cultured for $48 \mathrm{~h}$ according to the experimental requirements. The cells of different treatment groups were collected and centrifuged for $10 \mathrm{~min}$ at $1500 \mathrm{rpm}$. The supernatant was discarded and the cells were washed twice with $1 \times$ PBS solution. The cell density was adjusted to $2 \times 10^{6}$ cells per group. After centrifuged and discarded the supernatant, $70 \%$ ethanol after pre-cooling was added and incubated at $-20^{\circ}$ overnight. Then centrifuged at $2000 \mathrm{rpm}$ for $5 \mathrm{~min}$, discard the supernatant, wash it with PBS twice and resuspend, propidium iodide (PI) solution was added and avoid light reaction for $30 \mathrm{~min}$ at room temperature. It was detected by flow cytometry within $1 \mathrm{~h}$.

\section{Apoptosis:}

The cells in the logarithmic growth phase were prepared into a single cell suspension and inoculated in a six-well plate. After the cells adhered to the wall, different treatment drugs were added and cultured for $48 \mathrm{~h}$ according to the experimental requirements. The cells were digested and collected with trypsin without ethylenediamine tetraacetic acid (EDTA) to make cell suspension. The cells were washed with PBS twice and centrifuged at $2000 \mathrm{rpm}$ for $5 \mathrm{~min}$ to ensure that there were at least $(1-5) \times 10^{5}$ cells. After the cells were suspension by $500 \mu \mathrm{l}$ binding buffer, $5 \mu \mathrm{l}$ annexin V-fluorescein isothiocyanate (FITC) solution was added to mix well and finally $5 \mu 1$ PI was added at room temperature for 5-15 min. It was detected by flow cytometry within $1 \mathrm{~h}$.

\section{Detection of transwell migration and invasion:}

To detect the degree of cell migration and invasion, transwell was measured. The membranes coated with matrix glue (invasion assay) or matrix glue-uncoated (migration assay) were placed in the upper chamber overnight $\left(37^{\circ}\right)$. After trypsin cleavage, the serum-free medium was used to prepare the diluent. Next, the cells were transferred to the upper chamber and the medium supplemented with $5 \mathrm{mg} / \mathrm{l}$ fibronectin and $10 \%$ FBS was added to the lower chamber. After being cultured in a $5 \% \mathrm{CO}_{2}$ incubator $\left(37^{\circ}\right)$ for $24 \mathrm{~h}$, the cells entering the lower chamber through the membrane were fixed with methanol for about $20 \mathrm{~min}$ and then stained with crystal violet $(0.1 \%)$ for 10 min. Finally, the cells were examined and counted by a light microscope (Nikon, Japan).

\section{Quantitative real-time polymerase chain reaction detection:}

Michigan Cancer Foundation-7 (MCF7) cells cultured for $48 \mathrm{~h}$ in each group were collected and ribonucleic acid (RNA) was extracted by RNA extraction kit. The amplification primers of SOX2, MAP4K4, JNK, p53, TNF- $\alpha$ and glyceraldehyde 3-phosphate dehydrogenase (GAPDH) were designed and synthesized by TaKara company. RNA was reverse transcribed into complementary DNA by the PrimeScript real time (RT) kit according to the instructions, and then used for quantitative polymerase chain reaction (qPCR) according to the instructions of the SYBR Premix ExTaq TMII kit. qPCR was detected in ABIPRISM 7300 system with GAPDH as the internal reference, the relative expression levels of SOX2, MAP4K4, JNK, p53 and TNF- $\alpha$ were calculated. The experiment was repeated 3 times in each group and 3 samples were taken each time.

\section{Western blotting (WB):}

The total protein extracted by lysis buffer and determined by radioimmunoprecipitation and separated by sodium dodecyl sulfate polyacrylamide gel electrophoresis (SDS-PAGE) (Bio-Rad, USA). The protein was transfer to the polyvinylidene fluoride membrane and blocked with $5 \%$ skim milk for $1 \mathrm{~h}$. The primary antibody against p53 and Bcl-2-associated X protein (Bax) was incubated overnight at $4^{\circ}$. Next, after incubation with antiphospholipid syndrome (APS) immunoglobulin G (IgG)-horseradish peroxidase (HRP) (BOSTER) secondary antibody, the immune complex was displayed by enhanced chemiluminescence. The relative protein expression was determined by optical densitometry with Image $\mathbf{J}$ software.

\section{Immunohistochemical:}

The expressions of SOX 2 and MAP4K 4 in breast cancer tissues and adjacent normal tissues were detected by the immunohistochemical method. All tissues were fixed with $4 \%$ neutral formaldehyde solution and embedded with paraffin to make slices, followed by drying, dewaxing and hydration with xylene and gradient ethanol and antigen repair with citrate buffer solution. The primary antibody was added and incubated overnight at $4^{\circ}$ then reheated at $37^{\circ}$. The secondary antibody was added and incubated at room temperature for 30 min then rinsed repeatedly with PBS for 3 times 
and coloration by 3,3'-Diaminobenzidine (DAB) for 2 min. After rinsing with distilled water, it was stained with hematoxylin, dehydrated with gradient ethanol and xylene, sealed with neutral gum and observed under a light microscope. Ten visual fields (400x) were randomly selected and scored according to the degree of cell staining and the proportion of positive cells 6 . The degree of cell staining was 0 (no staining), 1 (weakly positive staining), 2 (moderate positive staining) and 3 (strong positive staining). The proportion of positive cells: 1 point $(<25 \%), 2$ points $(25 \%-50 \%), 3$ points $(50 \%-75 \%)$ and 4 points ( $\geq 75 \%)$. Taking the product of staining degree score and positive cell proportion score as the final result, the total score $<6$ was regarded as negative and vice versa.

\section{Statistical analysis:}

All the experiments were repeated three times independently. Quantitative data were expressed as mean \pm SD, GraphPad Prism 8.0.1 (GraphPad Software, La Jolla, CA) for comparing and evaluating data between groups. The differences between the two groups were analyzed by t-test. Kruskal-Wallis test was used to compare the differences among the three groups. $\mathrm{p}<0.05$ indicates that the difference is significant.

\section{RESULTS AND DISCUSSION}

The positive rates of SOX2 and MAP4K4 in breast cancer tissues were $83.33 \%$ and $86.67 \%$ respectively. The expression of SOX 2 and MAP4K 4 protein in breast cancer was not related to tumor diameter, patient's age and menopausal status but related to TNM stage and metastasis of the tumor, as shown in Table 1. The results of quantitative reverse transcription polymerase chain reaction (qRT-PCR) and WB showed that there was no significant difference in the expression of SOX2 messenger RNA (mRNA) and protein between the NC group and the control group. Compared with the $\mathrm{NC}$ group, the expression of SOX2 mRNA and protein in the si-SOX2 group decreased significantly, which indicated that we silenced the expression of the SOX2 gene (fig. 1). The results of qRT-PCR and WB showed that there were significant differences in the expression of mRNA and protein between the different groups $(\mathrm{p}<0.05)$. There was no significant difference between the $\mathrm{NC}$ group and the control group ( $\mathrm{p}>0.05)$. Compared with the NC group, the expression levels of mRNA and protein in the pcDNA-SOX2 group were significantly increased, while those in the si-SOX2 group were significantly decreased. Compared with the si-SOX2 group, the expression level of mRNA and protein in the si-SOX2+MAP4K4 group were increased (fig. 2).

TABLE 1: THE RELATIONSHIP BETWEEN THE PROTEIN EXPRESSION OF SOX2 AND MAP4K4 AND THEIR CLINICOPATHOLOGICAL FEATURES IN BREAST CANCER

\begin{tabular}{|c|c|c|c|c|c|}
\hline \multirow{2}{*}{$\begin{array}{c}\text { Clinicopathological } \\
\text { features }\end{array}$} & \multirow{2}{*}{ Cases } & \multicolumn{2}{|c|}{ SOX2 positive expression } & \multicolumn{2}{|c|}{ MAP4K4 positive expression } \\
\hline & & $\mathrm{n}$ & $\mathrm{p}$ & $\mathrm{n}$ & $\mathrm{p}$ \\
\hline \multicolumn{6}{|l|}{ Age $(y)$} \\
\hline$<45$ & 12 & 8 & \multirow[t]{2}{*}{$>0.05$} & 7 & \\
\hline$>45$ & 48 & 42 & & 45 & $>0.05$ \\
\hline \multicolumn{6}{|l|}{ Menopausal status } \\
\hline Yes & 34 & 23 & \multirow[t]{2}{*}{$>0.05$} & 22 & \multirow{2}{*}{$>0.05$} \\
\hline No & 26 & 14 & & 15 & \\
\hline \multicolumn{6}{|l|}{ Tumor diameter } \\
\hline$>2 \mathrm{~cm}$ & 33 & 26 & \multirow[t]{2}{*}{$>0.05$} & 26 & \multirow{2}{*}{$>0.05$} \\
\hline$\leq 2 \mathrm{~cm}$ & 27 & 20 & & 18 & \\
\hline \multicolumn{6}{|l|}{$\begin{array}{l}\text { Lymphatic } \\
\text { metastasis }\end{array}$} \\
\hline Yes & 24 & 22 & $<0.05$ & 20 & \multirow{2}{*}{$<0.05$} \\
\hline No & 36 & 10 & & 11 & \\
\hline \multicolumn{6}{|l|}{ TNM stage } \\
\hline$|-| I$ & 25 & 13 & \multirow[t]{2}{*}{$<0.05$} & 12 & \multirow{2}{*}{$<0.05$} \\
\hline III-IV & 35 & 28 & & 31 & \\
\hline
\end{tabular}




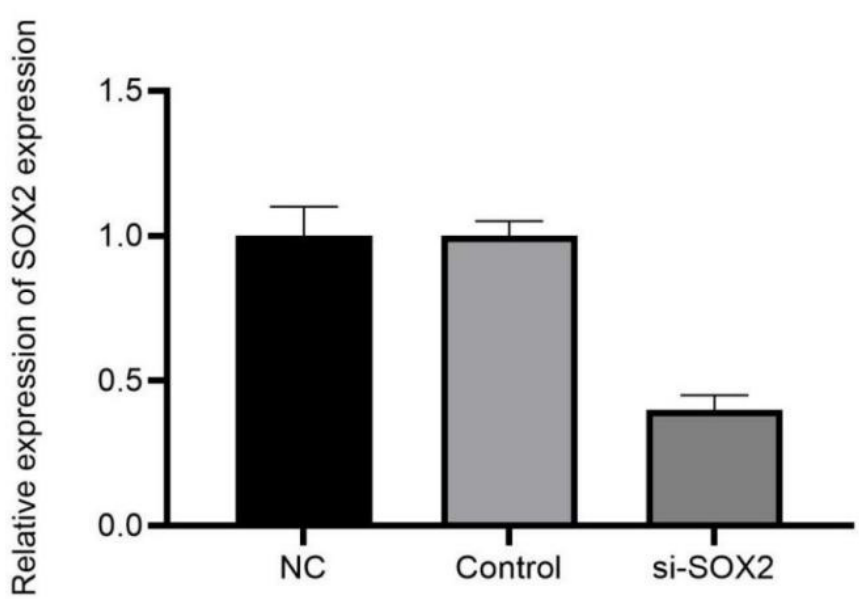

Fig. 1: SOX2 mRNA expression after SOX2 gene silencing, ( $\square$ )-NC; ( $\square$ )-Control, ( $\square$ )-si-SOX2

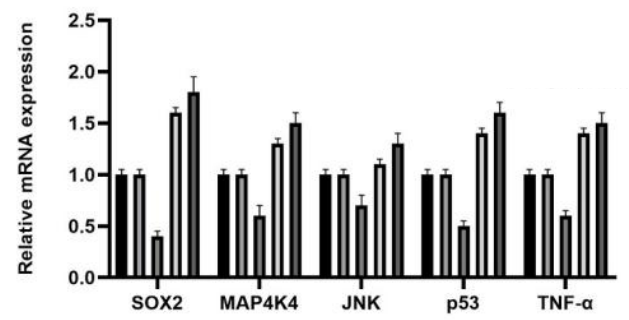

B

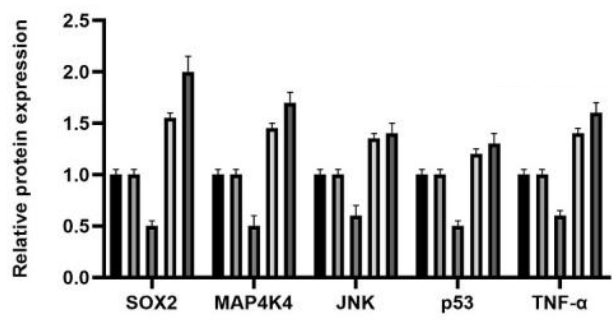

Fig. 2: Effect of the SOX2 gene silencing on MAP4K4 signal pathway; ( $\square$ ) NC; ( $($ ) ) Control; ( $\square$ ) si-SOX2; ( ロ ) si-SOX2+MAP4K4; ( ロ ) pcNDA-SOX2

CCK8 detection showed that there was no significant difference in the ability of cell proliferation in each group at $0 \mathrm{~h}(\mathrm{p}>0.05)$, and there was a significant difference between 24-96 h. There was no significant difference between the blank group and $\mathrm{NC}$ group $(\mathrm{p}>0.05)$. Compared with the NC group, the cell proliferation rate in the pcDNA-SOX2 group was significantly increased at 48-96 h, while that in the si-SOX2 group was significantly decreased at 24-96 h. Compared with the si-SOX2 group, the cell proliferation rate of the siSOX2+MAP4K4 group increased gradually at 24-96 $\mathrm{h}$ (fig. 3). The results of the scratch test and the Transwell invasion showed that there were significant differences in cell migration and invasion between groups. There was no significant difference between the blank group and $\mathrm{NC}$ group ( $>>0.05)$. Compared with the $\mathrm{NC}$ group, the ability of cell migration and invasion in the pcDNASOX2 group was significantly enhanced, while that in the si-SOX2 group was significantly decreased. Compared with the si-SOX2 group, the ability of cell migration and invasion in si-SOX2+MAP4K4 group was decreased (fig. 4).
The results of flow cytometry showed that there was a significant difference in the apoptosis between the groups $(p<0.05)$. There was no significant difference between the blank group and $\mathrm{NC}$ group ( $>00.05)$. Compared with the $\mathrm{NC}$ group, apoptosis was significantly decreased in the pcDNA-SOX2 group, but significantly increased in the si-SOX2 group. The ability of apoptosis in siSOX2+MAP4K4 group was lower than that in the siSOX2 group (fig. 5). Breast cancer mainly refers to the malignant tumor that occurs in the epithelial tissue of the mammary gland. Because the breast was not the main organ to maintain life, breast cancer in situ was not fatal ${ }^{[7]}$. However, breast cancer may metastasize to the liver, kidney and other important tissues or organs, which affecting the life safety of patients. The incidence of breast cancer in China was increasing. In recent years, molecular biology technology has become a research hotspot in the diagnosis and treatment of breast cancer ${ }^{[8]}$. 


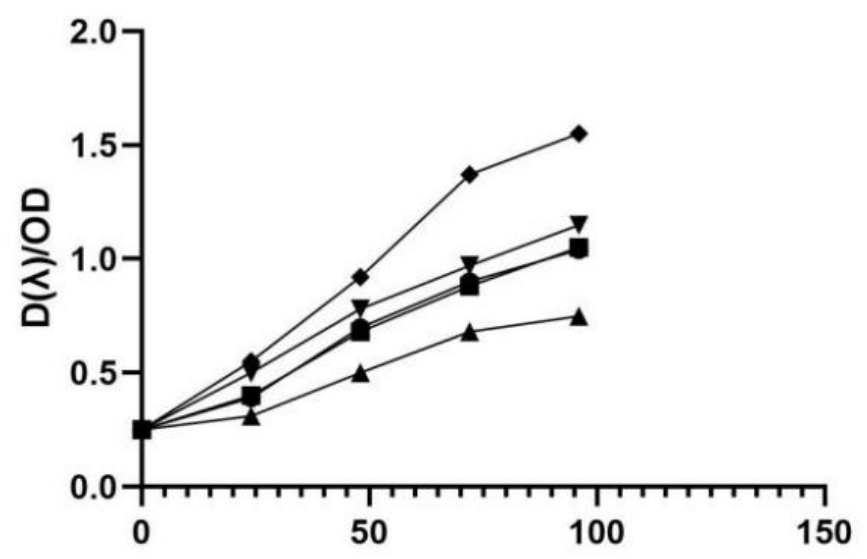

Fig. 3: Effect of the SOX2 gene silencing on cell proliferation; $(+\bullet) \mathrm{NC}$; $(\rightarrow)$ Control; $(\rightarrow-)$ si-SOX2; $(\rightarrow)$ si-SOX2+MAP4K4; ( $\rightarrow$ ) pcNDA-SOX2
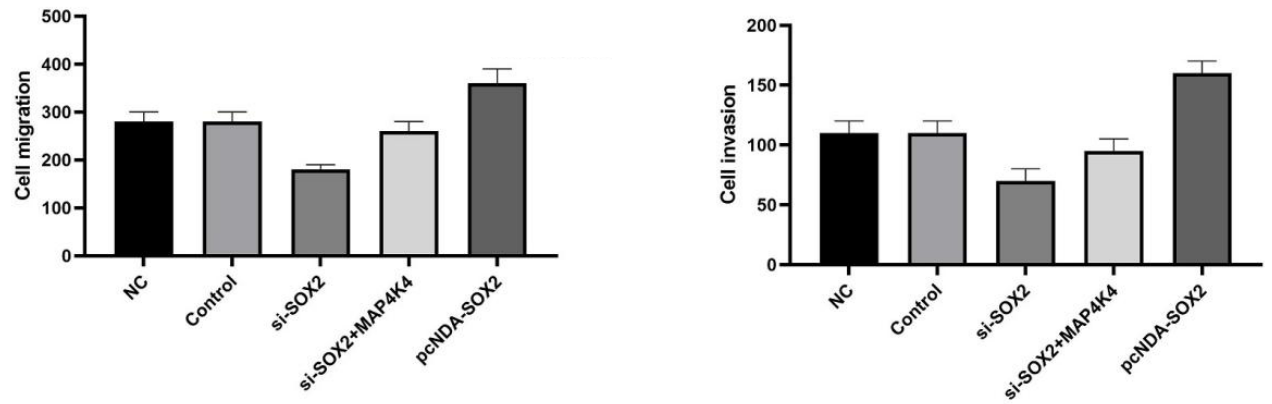

Fig. 4: Effects of the SOX2 gene silencing on cell migration and invasion; ( $\square$ ) NC; ( $\square$ ) Control; ( $\square$ ) si-SOX2; (ㅁ) si-SOX2+MAP4K4; ( $\square$ ) pcNDA-SOX2

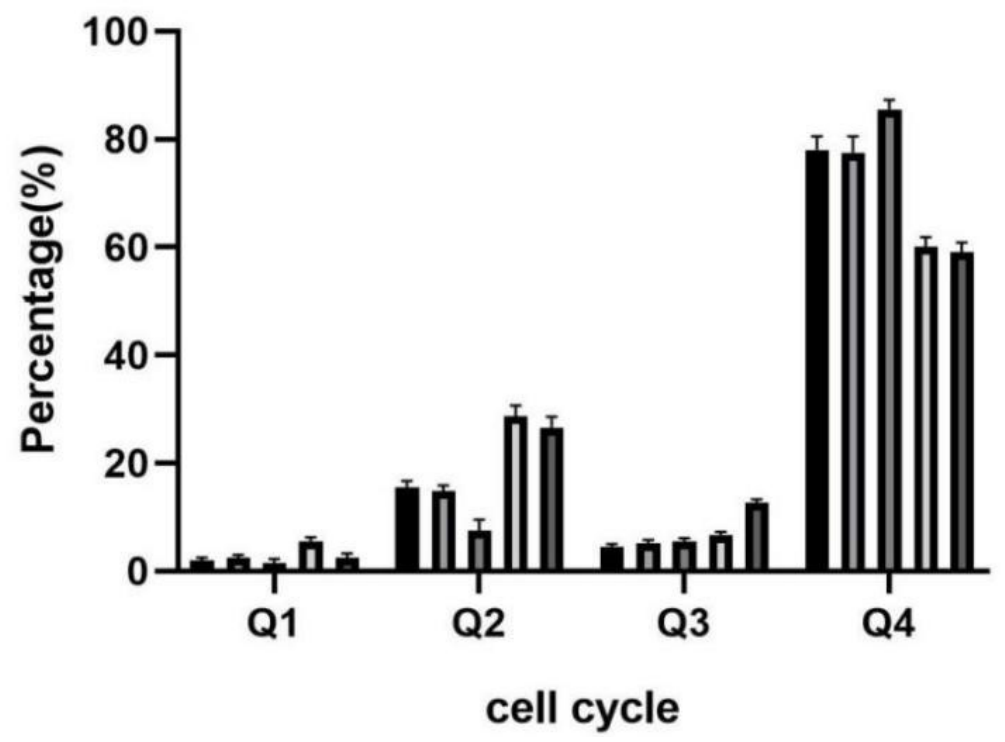

Fig. 5: Effect of the SOX2 gene silencing on apoptosis; ( $\square$ ) NC; ( $(\square)$ Control; ( $\square$ ) si-SOX2; (口) si-SOX2+MAP4K4; ( $\square$ ) pcNDA-SOX2 
SOX2 was an important member of the SOX family. It was expressed in the inner cell mass, ectoderm and germ cells of the early embryo which was closely related to the growth and development of the ectoderm in the early stage of the embryo. Knockout of SOX2 will lead to the differentiation of the trophoblast of ESCs, the expression deletion of SOX2 will make the embryo stop at the implantation stage, and the expression of SOX2 strictly follows the time and space restriction ${ }^{[10]}$. Tripolitsioti et al. ${ }^{[11]}$ confirmed that the $\mathrm{SOX} 2$ was highly expressed in glandular intraepithelial neoplasia and pancreatic ductal carcinoma. Nidhi et al ${ }^{[12]}$ studies have shown that the SOX2 was highly expressed in breast cancer. In this study, the expression of SOX2 and MAP4K4 in the breast cancer tissues (63.33\%) was significantly higher than those in normal tissues (13.33 $\%$ ), indicating that SOX2 and MAP4K4 may play a key role in the formation, occurrence and development of breast cancer. The expression of SOX2 and MAP4K4 in breast cancer was not related to the tumor size, menopausal state and age but related to the TNM stage of the tumor, indicating that the expression of SOX2 and MAP4K4 was related to the malignant biological behavior of breast cancer. At the same time, the results showed that SOX2 was closely related to lymph node metastasis $(p<0.05)$. The results suggest that the sustained high expression of SOX2 and MAP4K4 may further participate in the invasion and growth of breast cancer. Due to the up-regulated expression of SOX2 and MAP4K4 in breast cancer, normal cells lose the limitation of apoptosis in the proliferation cycle. The continuous proliferation and division of breast cancer cells reduce the number of apoptosis, resulting in the formation of metastatic tumors in adjacent and distant tissues of malignant tumors. The results show that the SOX2 and MAP4K4 may play an important role in lymph node metastasis of breast cancer.

A large number of studies have shown that SOX2 was involved in the apoptosis, but the mechanism of SOX2 in the apoptosis pathway was not clear ${ }^{[13-16]}$. It has been found that SOX2 gene silencing activates the death receptor and mitochondrial signal transduction pathway and effectively induces the apoptosis in human non-small cell lung cancer. It was worth noting that the down-regulation of SOX2 leads to the activation of MAP4K4, which was related to the cell apoptosis. Therefore, this study speculates that there may be SOX2 in breast cancer that inhibits the expression of downstream related proteins of MAP4K4 through the MAP4K4 signal pathway and blocks the development of breast cancer. Based on this corollary, this paper verified the mechanism of the SOX2 gene silencing mediated MAP4K4 signal pathway on the biological characteristics of breast cancer cells.

The results of this study show that SOX2 silencing can reduce the mRNA and protein expression of the SOX2 gene significantly. At the same time, the expression of SOX2, MAP4K4, JNK, p53 and TNF- $\alpha$ increased after pcDNA-SOX2 transfection, while decreased after siSOX2 transfection. Therefore, compared with the effect of the overexpression of SOX2 on the expression of proteins related to MAP4K4 signal pathway in cancer cells, SOX2 silencing can activate the MAP4K4 signal pathway. In addition, a si-SOX2+MAP4K4 group was established to support the activation of MAP4K4 signal pathway by silencing SOX2 expression. The expression of SOX2, JNK, p53, and TNF- $\alpha$ was inhibited by the transfection of si-SOX2 and MAP4K4, which confirmed the rationality of this conjecture. At the same time, si-SOX2 transfection led to the weakening of cell growth, the weakening of cell proliferation, migration and invasion, and the increase of apoptosis. Si-SOX2+MAP4K4 transfection can reverse the above trend after si-SOX2 transfection. Therefore, SOX2 gene silencing can activate the MAP4K4 signal pathway and participate in the regulation of the biological characteristics of breast cancer cells.

In conclusion, this study confirmed that SOX2 silencing can promote breast cancer cell proliferation, migration and invasion and inhibit apoptosis by activating MAP4K4 signal pathway, which provides a potential molecular target for targeted therapy of breast cancer.

\section{Acknowledgements:}

This work was supported by the Medical Science and Technology Development Project of Nanjing (YKK16237), the Clinical Medicine Science and Technology Development Fund Project of Jiangsu University (JLY20180033) and the Medical Science and Technology Development Special Fund Project of Nanjing (YKK19136).

\section{Conflicts of interest:}

The authors declared no conflict of interest.

\section{REFERENCES}

1. Xiao W, Zheng S, Xie X, Li X, Zhang L, Yang A, et al. SOX2 promotes brain metastasis of breast cancer by upregulating the expression of FSCN1 and HBEGF. Mol Ther Oncolytics 2020;17:118-29.

2. El-Benhawy SA, El-Sheredy HG, Ghanem HB, Abo El-Soud AA. Berberine can amplify cytotoxic effect of radiotherapy by targeting cancer stem cells. Breast Cancer Manag 2020;9(2):BMT41. 
3. Deng $\mathrm{Y}$, Chen D, Gao F, Lv H, Zhang G, Sun X, et al. Silencing of long non-coding RNA GAS5 suppresses neuron cell apoptosis and nerve injury in ischemic stroke through inhibiting DNMT3B-dependent MAP4K4 methylation. Transl Stroke Res 2020;11(5):950-66.

4. Kim JW, Berrios C, Kim M, Schade AE, Adelmant G, Yeerna $\mathrm{H}$, et al. STRIPAK directs PP2A activity toward MAP4K4 to promote oncogenic transformation of human cells. eLife 2020;9:e53003.

5. Watts ME, Wu C, Rubin LL. Suppression of MAP4K4 signaling ameliorates motor neuron degeneration in amyotrophic lateral sclerosis-molecular studies toward new therapeutics. J Exp Neurosci 2019;13:1179069519862798.

6. Tang W, Zhang X, Tan W, Gao J, Pan L, Ye X, et al. miR-145$5 \mathrm{p}$ suppresses breast cancer progression by inhibiting SOX2. J Surg Res 2019;236:278-87.

7. Wang F, Zhao L, Zhang J, Meng Z, Zhou C, Wang G, et al. Chemotherapy-induced miR-141/MAP4K4 signaling suppresses progression of colorectal cancer. Biosci Rep 2018;38(6):BSR20180978.

8. Peng HY, Liang YC, Tan TH, Chuang HC, Lin YJ, Lin JC. RBM4a-SRSF3-MAP4K4 splicing cascade constitutes a molecular mechanism for regulating brown adipogenesis. Int J Mol Sci 2018;19(9):2646.

9. Zhang JM, Wei K, Jiang M. OCT4 but not SOX2 expression correlates with worse prognosis in surgical patients with triplenegative breast cancer. Breast Cancer 2018;25(4):447-55.

10. Liu P, Tang H, Song C, Wang J, Chen B, Huang X, et al. SOX2 promotes cell proliferation and metastasis in triple negative breast cancer. Front Pharmacol 2018;9:942.
11. Tripolitsioti D, Kumar KS, Neve A, Migliavacca J, Capdeville $\mathrm{C}$, Rushing EJ, et al. MAP4K4 controlled integrin $\beta 1$ activation and c-Met endocytosis are associated with invasive behavior of medulloblastoma cells. Oncotarget 2018;9(33):23220.

12. Gupta N, Gopal K, Wu C, Alshareef A, Chow A, Wu F, et al. Phosphorylation of Sox 2 at threonine 116 is a potential marker to identify a subset of breast cancer cells with high tumorigenecity and stem-like features. Cancers 2018;10(2):41.

13. Liu K, Xie F, Gao A, Zhang R, Zhang L, Xiao Z, et al. SOX2 regulates multiple malignant processes of breast cancer development through the SOX2/miR-181a-5p, miR-30e-5p/ TUSC3 axis. Mol Can 2017;16(1):1-4.

14. Kamarlis RK, Lubis MN, Hernowo BS, Kar AS. Immunoexpression of P63 and SOX2 in triple-negative breast cancers, Indonesia. F1000Res 2017;6:1780.

15. Leung EY, Askarian-Amiri ME, Sarkar D, Ferraro-Peyret C, Joseph WR, Finlay GJ, et al. Endocrine therapy of estrogen receptor-positive breast cancer cells: early differential effects on stem cell markers. Front Oncol 2017;7:184.

16. Jin M, Chu H, Li Y, Tao X, Cheng Z, Pan Y, et al. MAP4K4 deficiency in CD4+ T cells aggravates lung damage induced by ozone-oxidized black carbon particles. Environ Toxicol Pharmacol 2016;46:246-54.

This is an open access article distributed under the terms of the Creative Commons Attribution-NonCommercial-ShareAlike 3.0 License, which allows others to remix, tweak, and build upon the work non-commercially, as long as the author is credited and the new creations are licensed under the identical terms

This article was originally published in a special issue,

"Therapeutic Perspectives in Biomedical Research and Pharmaceutical Sciences and their Nursing Methods"

Indian J Pharm Sci 2021:83(4)Spl issue "82-89" 